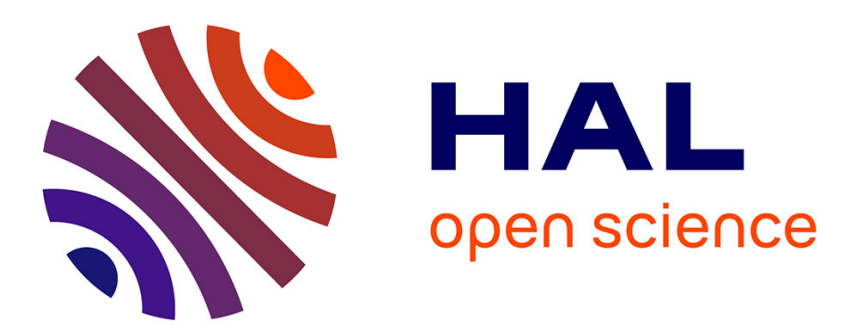

\title{
Exchange coupling mechanism for magnetization reversal and thermal stability of Co nanoparticles embedded in a $\mathrm{CoO}$ matrix
}

\author{
Dominique Givord, Vassil Skumryev, Josep Nogués
}

\section{To cite this version:}

Dominique Givord, Vassil Skumryev, Josep Nogués. Exchange coupling mechanism for magnetization reversal and thermal stability of Co nanoparticles embedded in a $\mathrm{CoO}$ matrix. Journal of Magnetism and Magnetic Materials, 2005, pp.111. hal-00102531

\section{HAL Id: hal-00102531 \\ https://hal.science/hal-00102531}

Submitted on 10 Nov 2006

HAL is a multi-disciplinary open access archive for the deposit and dissemination of scientific research documents, whether they are published or not. The documents may come from teaching and research institutions in France or abroad, or from public or private research centers.
L'archive ouverte pluridisciplinaire $\mathbf{H A L}$, est destinée au dépôt et à la diffusion de documents scientifiques de niveau recherche, publiés ou non, émanant des établissements d'enseignement et de recherche français ou étrangers, des laboratoires publics ou privés. 


\title{
Exchange coupling mechanism for magnetization reversal and thermal stability of Co nanoparticles embedded in a $\mathrm{CoO}$ matrix
}

\author{
Dominique Givord $^{a}$, Vassil Skumryev ${ }^{b, *}$, Josep Nogués ${ }^{b}$ \\ ${ }^{a}$ Laboratoire Louis Néel, CNRS, 38042-Grenoble-cedex 9, France \\ ${ }^{b}$ Institució Catalana de Recerca i Estudis Avançats (ICREA) and Department de Física, Universitat Autònoma de Barcelona, 08193 \\ Bellaterra, Spain
}

Elsevier use only: Received date here; revised date here; accepted date here

\begin{abstract}
A model providing a semi-quantitative account for the magnetic behavior of Co nanoparticles embedded in a CoO matrix is presented. The results confirm that exchange coupling at the interface between FM and AFM nanostructures, could provide an extra source of magnetic anisotropy, leading to thermal stability of the FM nanoparticles. It is shown that perpendicular coupling between the AFM and FM moments may result in large coercivities. The energy barrier, which works against reversal is due to the AFM susceptibility anisotropy. Experimentally observed exchange-bias is tentatively ascribed to preexisting intrinsic canting of the AFM moments at the interface.
\end{abstract}

(C) 2010 Elsevier B.V. All rights reserved

PACS: 75.50.Tt; 7530.Gw; 75.70.Cn

Keywords: Superapramagnetism, exchange bias, magnetic nanoparticles, antiferromagnets

\section{Introduction}

Today's interest in nanoparticle magnetism is stimulated by a variety of potential applications, ranging from soft to hard magnetic materials and from ultra-high density information storage to medicine [1]. Thermal stability of the nanoparticle magnetization is one of the key issues for most of applications and is particularly critical for magnetic recording [2]. Several experimental studies [3-7] have recently indicated that exchange coupled ferromagnetic (FM) and antiferromagnetic (AFM) nanostructures experience an improved thermal stability, as it has been theoretically suggested $[9,10]$.

We have recently reported an increase of the superparamagnetic blocking temperature of almost two orders of magnitude for Co nanoparticles (3-4 nm in diameter) embedded in AFM $\mathrm{CoO}$ matrix [3]. The studied nanostructure exhibits all the features of exchange bias systems [11]: loop shift, in the field axis, of $\mu_{o} H_{E B}=0.74 \mathrm{~T}$ and enhanced coercivity of $\mu_{o} H_{C}=0.76 \mathrm{~T}$ at $4.2 \mathrm{~K}$. In ref. 3 , we briefly introduced a model which provided a semiquantitative account for the observed behavior, thus confirming that exchange coupling at the interface between FM and AFM nanostructures, could provide an extra source of magnetic anisotropy, leading to thermal stability of the FM nanoparticles.

Here we present a more detailed description of the proposed model. Comparison with experimental data suggests that the so-called perpendicular coupling mechanism, instead of the usual parallel coupling, is the

\footnotetext{
*Corresponding author. Tel.: +34 935811 657; fax: +34935811350

E-mail address: vassil.skumryev@uab.es (Vassil Skumryev)
} 
dominant mechanism involved in this type of nanostructures. The proposed model can be used when discussing exchange coupled FM/AFM nanostructures, similar to the one described here.

\section{The Model}

\subsection{Basic parameters}

Based on Meiklejohon and Bean rigid spin model, the classical expression of the bias field, $H_{E B}$, can be written as [12]:

$$
H_{E B}=J_{e x} S_{F M} S_{A F M} / A M_{F M} t_{F M}
$$

where $S_{F M}$ and $S_{A F M}$ are the individual FM and AFM spins, $M_{F M}$ is the ferromagnetic magnetization, $t_{F M}$ is the ferromagnetic layer thickness, and $A$ is the surface area over which the exchange term is calculated. In $\mathrm{CoO}$, the total Co moment is $3.8 \mu_{\mathrm{B}}$ and the orbital-to-spin ratio, $\mathrm{L} / \mathrm{S}$ $=0.95$, from which the AFM spin moment $\mu_{A F M}=2.55 \mu_{\mathrm{B}}$ is derived. The parameter $J_{e x} S_{F M} S_{A F M}$, may be re-expressed as $\mu_{0} \mu_{A F M} H_{\text {exch }}$, where $H_{\text {exch }}$ is an effective exchange field created by the ferromagnetic spin moments. For $\mathrm{Co} / \mathrm{CoO}$, from the accepted value of $J_{e x} S_{F M} S_{A F M}=143 \mathrm{~K}$ [13], $\mu_{0} H_{\text {exch }}=84 \mathrm{~T}$ can be derived (see App. A).

\subsection{Parallel versus perpendicular coupling}

A priori, two possible coupling mechanisms may be envisaged between the FM and the AFM moments at the interface.

(i) Usually, the FM moments are assumed coupled parallel to the uncompensated AFM moments. In principle, uncompensated moments (in otherwise compensated AFM structure) could arise only on a flat surface, which is perpendicular to the propagation vector of the antiferromagnetic structure. In the present case, the FM Co nanoparticles can be viewed as embedded inside small cavities of an AFM CoO single crystal (see Fig.1). There is no reason for the AFM side of the interface to show strong uncompensation, except the one due to statistics. Considering that the surface per $\mathrm{Co}$ atom in the $\mathrm{CoO}$ structure is $0.072 \mathrm{~nm}$ [14]. and assuming that the $\mathrm{CoO}$ shell at the surface of the metal core has an average diameter of $4.2 \mathrm{~nm}$ it is deduced that there are approximately $750 \mathrm{Co}$ atoms at the AFM interfaces and the unbalance should be of the order of $\sqrt{750}=27$ atoms $\approx 4 \%$ of the total number of atoms. The interface coupling energy between the AFM and FM magnetization may thus be expressed as - 0.04 $\mu_{0} H_{\text {exch }} M^{\prime}$ where $M^{\prime}$ is the AFM magnetization, which is derived from $\mu_{A F M}$ and amounts to $0.76 \mathrm{~T}$. The interface coupling energy, normalized to the volume, $V_{A F M}\left(V_{A F M}=\right.$ $10^{-26} \mathrm{~m}^{3}$ ), of one $\mathrm{CoO}$ atomic layer at the interface with the Co core, it amounts to approximately $-0.210^{7} \mathrm{~J} / \mathrm{m}^{3}$. (ii) Another mechanism may also be envisaged, which does not require un-compensation to be invoked. At a compensated interface (Fig.1a), the number of AFM moments from each sublattice is the same, the resulting magnetisation is zero and thus the coupling vanishes to first order. To second order, the FM moments couple perpendicular to the AFM moments (Figures $1 \mathrm{~b}$ and 2a), the exchange field induces a canting of the AFM moments along the direction denoted $z$ and a ferromagnetic component is induced along the FM Co moments [15-16]. Considering that $H_{\text {exch }}$ is formally equivalent to an external field, the coupling energy can be written as [18] :

$$
E=(-1 / 2) \chi_{A F} \mu_{0} H_{e x c h}^{2}
$$

where $\chi_{\mathrm{AF}}$ is the interface AFM susceptibility. This expression is fully analogous to the classical expression describing the behaviour of an antiferromagnet in an applied magnetic field. The minimum energy state is given by the configuration between the FM and AFM moments, such that the AFM susceptibility is a maximum. In the case of $\mathrm{CoO}$, it may be assumed that there exists an easy magnetization plane, $\mathrm{P}_{\mathrm{AFM}}$, approximately perpendicular to the [111] difficult direction. The AFM moments are confined in this plane and the corresponding susceptibility may be called $\chi_{\perp}^{\max }$ (the superscript max indicates that the susceptibility is maximum within $\mathrm{P}_{\mathrm{AFM}}$ ). This $\chi_{\perp}^{\max }$ could be expressed [17] as

$$
\chi_{\perp}^{\max }=1 / w(1+\alpha) \quad \text { with } \quad \alpha=K_{1} / 2 w \mu_{0} M^{\prime 2}
$$

where $w$ is the molecular field coefficient between AFM sublattices, and $K_{l}$ is the second order anisotropy constant, relative to the anisotropy difference between the AFM axis and the direction along which $H_{\text {exch }}$ is induced.

The parameter values used in the calculation were $w=$ 100 (see App. B) for the surface intersublattice molecular field coefficient and $K_{l}=2.710^{7} \mathrm{~J} / \mathrm{m}^{3}$ (in the absence of experimental data for the $\mathrm{CoO}$ anisotropy constant, we took the theoretical value derived by Kanamori [19]). One obtains $2 w \mu_{0} M^{, 2}=0.910^{7} \mathrm{~J} / \mathrm{m}^{3}$, thus $\alpha=0.3$ and $E=$ $2.110^{7} \mathrm{~J} / \mathrm{m}^{3}$. The canting angle $\theta$ is thus estimated to about $24^{\circ}$. The coupling energy, in the perpendicular coupling case, is one order of magnitude larger than the one due to un-compensation, calculated above in the case of parallel coupling.

\subsection{Magnetization Reversal and Coercivity}

Qualitatively, the experimentally observed large values of coercive field $H_{C}$ (and bias field $H_{E B}$ ) may be ascribed to the fact that interface effects (i.e., $H_{\mathrm{EB}} \propto 1 / \mathrm{t}_{\mathrm{FM}}$ in thin films) are relatively larger for such extremely small nanoparticles than they are for multilayers whose typical thickness is around $20 \mathrm{~nm}$. Additionally, the coercive field of small nanoparticles, which reverse their magnetisation by coherent rotation, is in general bigger than that of large systems where reversal is nucleated at defects. 
Quantitatively, the existence of a large coercivity in the present system may be ascribed to the fact that the antiferromagnetic susceptibility, $\chi_{\mathrm{AF}}$, in Eq. 2 is expected to show anisotropy and thus an energy barrier against reversal should exist.

To evaluate the strength of the coercive field, let us assume that a field is applied antiparallel to the initial magnetisation direction. FM magnetisation reversal may occur within $\mathrm{P}_{\mathrm{AFM}}$ or within the plane $\mathrm{P}$, which is perpendicular to the axis $x$ of easy AFM magnetisation and contains the difficult directions (Figures $2 b$ and $2 c$, respectively).

The energy barrier per unit volume, $\Delta E$, may be expressed as :

$$
\Delta E=(1 / 2) \Delta \chi_{\perp} \mu_{0} H_{e x c h}^{2}
$$

where $\Delta \chi_{\perp}$ is the susceptibilty anisotropy, i.e. the difference between the initial direction of maximum susceptibility and the direction of minimum susceptibility within the rotation plane.

In the case when the rotation occurs within $\mathrm{P}_{\mathrm{AFM}}$, (Fig. $2 b$ ), the susceptibility is a minimum when the FM moments are aligned along $x$ At $0 \mathrm{~K}, \chi_{\perp} \square$ vanishes along $x$, thus $\Delta \chi_{\perp}=\chi_{\perp}^{\max }=-7.610^{-3}$ and $\Delta E=2.110^{7} \mathrm{~J} / \mathrm{m}^{3}$ is obtained.

On the other hand when the rotation occurs within $\mathrm{P}$, (Fig. 2c), $\Delta \chi_{\perp}=-w^{-1} \alpha(1+\alpha)^{-1}=-2.310^{-3}$ and $\Delta E=6.4$ $10^{6} \mathrm{~J} / \mathrm{m}^{3}$ are derived. The energy barrier is 3 times smaller for this process than for rotation within $\mathrm{P}_{\mathrm{AFM}}$ and it is concluded that rotation occurs within $\mathrm{P}$ (, since the energy is lower.)

Taking into account that that $H_{A}=2 K / \mu_{0} M_{s}=2 \Delta E / \mu_{0} M_{s}$ and $H_{c}=0.5 H_{A}$, due to the random orientation of easy axis, the deduced coercive field is $\mu_{0} H_{c}=\Delta e_{\text {min }} / M_{s} V_{F M}$ where $\Delta e_{\min }=\Delta E_{\text {min }} V_{A F M}\left(\Delta E_{\text {min }}\right.$ is the minimum energy barrier per unit volume), $V_{F M}$ is the FM nanoparticle volume (33.5 $\mathrm{nm}^{3}$ ) and $M_{s}$ is the ferromagnetic spontaneous magnetization $\left(\mu_{0} M_{s}=1.8 \mathrm{~T}\right)$. Using these values $\mu_{0} H_{c}=$ $1.3 \mathrm{~T}$ is obtained. Considering the uncertainty in the values of most of these parameters, the agreement with the experimental value, $\mu_{0} H_{c}=0.76 \mathrm{~T}$, can be considered as very satisfactory.

It is worth mentioning that unlike usual thin film exchange-bias systems, calculated and experimental coercive field values are in fair agreement. Due to their very small size, the FM particles can be thought to be coupled to a unique AF domain and consequently there is no competition between different coupling terms.

As discussed in Ref. 3, this energy barrier also gives rise to an improvement of the thermal stability of the nanoparticles, which results in an enhancement of the superparamagnetic blocking temperature.

\subsection{Exchange Bias}

Exchange bias implies that the FM/AFM coupling energy differs in the initial and the final magnetization states of the Co FM nanoparticles. In the present model, the energies of these two states are equal (see Figures 2a and $2 d)$. Thus, the model cannot account for the existence of exchange bias in the $\mathrm{Co} / \mathrm{CoO}$ system.

Exchange bias is usually ascribed to uncompensation of the AFM magnetization [13, 16, 17] and we thus considered the possible contribution of such a term. As mentioned above, on the basis of statistical considerations, the uncompensated AFM magnetization is expected to be about $4 \%$. To first order, the FM moments align at an angle $\beta$ from $z$, such that $\operatorname{tg} \beta=\left(0.04 \operatorname{cotan} 24^{\circ}\right) / 2$, thus $\beta=2.6^{\circ}$. It may be assumed that after cooling under field, the FM moments are aligned at an angle $\beta$ from $z$ and that they reverse by $180^{\circ}$ under field. This provides an approximate value for the bias-field, $H_{E B}$, according to this process which is given by:

$\mu_{0} M_{s} H_{E B} V_{F M} \cong 0.04 \sin \beta \cos 24^{0} \mu_{0} M^{\prime} H_{e x c h} V_{A F M}$

The calculated bias field is two orders of magnitude smaller than the experimental one, i.e. this mechanism cannot be the source of the experimentally observed exchange bias.

An alternative mechanism for exchange bias may be suggested that requires that the AFM moments show an intrinsic canting i.e. different from the one induced by $\mu_{0} H_{\text {exch }}$. Such a canting is known to exist at the surface of magnetic oxide nanoparticles $[19,20]$, where it leads to the appearance of a ferromagnetic moment which is 5-10 times higher than the one expected from uncompensation. By analogy it may be expected to exist at the surface of small cavities.

Let $\theta$ ' be a phenomenological angle representative of the intrinsic canting. The bias field is given by :

$$
\mu_{0} M_{s} H_{E B} V_{F M}=J_{e x} S_{F M} S_{A F M} 2 \sin \theta^{\prime}
$$

From $\mu_{0} H_{E B}=0.74 \mathrm{~T}$, and the same values as above for other parameters, one obtains $\sin \theta^{\prime}=0.41$ and $\theta^{\prime}=24^{\circ}$. This is of the same order of magnitude as in magnetic oxide nanoparticles [21].

Note that as a result of this intrinsic canting, the coercive energy barrier discussed in section 2.2 , is reduced by a factor of approximately $\cos \theta^{\prime}(\approx 0.9)$. Consequently, the disagreement between the calculated and experimental coercive field is further reduced.

\section{Conclusions}

For Co nanoparticlees embedded in an AFM matrix, we have shown that perpendicular coupling between the AFM and FM moments may be the source of large coercivities. The energy barrier which works against reversal is due to the AFM susceptibility anisotropy. This energy barrier also 
results in an improvement of the thermal stability of the nanoparticles. Conversely, exchange-bias is tentatively ascribed to pre-existing canting of the AFM moments at the interface.

\section{Appendix A. Exchange field evaluation}

In the classical expression for the bias field, eq.1, the left-hand side term represents the Zeeman energy over the volume $A t_{F M}$, while the right-hand side term represents the interfacial exchange energy, over the surface area $A$, i.e., the exchange energy per unit area is :

$$
E_{e x c}=J_{e x} S_{F M} S_{A F M} / A
$$

To relate expressions (1) and (A1) to usual expressions for exchange energy, let the exchange energy between 2 spins $S_{F M}$ and $S_{A F M}$ be expressed as :

$$
e_{F M-A F M}=2 J S_{F M} S_{A F M}
$$

where $J$ is the exchange integral. Considering that a given AFM spin has $z_{A F M-F M}$ FM neibghours, and the exchange energy per AFM spin is :

$$
e_{A F M}=2 z_{A F M-F M} J S_{F M} S_{A F M} .
$$

from which one derives the energy per unit surface area :

$$
E_{e x c h}=\left(2 / A_{A F M}\right) z_{A F M-F M} J S_{F M} S_{A F M}
$$

where $A_{A F M}$ is the surface area occupied by one Co AFM atom.

In the detailed calculation [13], the interfacial exchange energy $U_{i}$ over the crystallite $i$ is obtained by summing over the $N$ AFM interfacial atoms. This implies that the calculations were performed by using the AFM atoms as reference, as done in the present discussion. Thus, equations (5) and (2) lead to :

$$
\left(2 / A_{A F M}\right) z_{A F M-F M} J=J_{e x} / A_{A F M}
$$

Thus $J_{e x}$ in (2) is related to the usual exchange integral $J$ through :

$$
J_{e x}=2 z_{A F M-F M} J
$$

The exchange field on a given AFM moment is obtained from :

$$
J_{e x} S_{F M} S_{A F M}=\mu_{A F M} \mu_{0} H_{e x c h}
$$

From $J_{e x} S_{C o} S_{C o O}=143 \mathrm{~K}[13]$ and from $\mu_{A F M}=2.55 \mu_{\mathrm{B}}$ (spin part of the moment only, see appendix B). $\mu_{0} H_{\text {exch }}=84 \mathrm{~T}$ is derived.

\section{Appendix B. Molecular field coefficients evaluation}

Within the molecular field formalism, two molecular field coefficients may be defined, $w$ and $w^{\prime}$, which represent the coupling energy within a given sublattice and between sublattices respectively. $\mathrm{w}$ and $\mathrm{w}$ ' are related to $\mathrm{T}_{\mathrm{N}}$ through $w+w^{\prime}=3 k T_{N} / N^{\prime} \mu_{0} \mu_{\text {eff }}^{2}$, where $N^{\prime}$ is the number of atoms per unit volume within a given sublattice and $\mu_{\text {eff }}$ is the antiferromagnetic Co effective moment. The Néel temperature and lattice parameter for $\mathrm{CoO}$ are $\mathrm{T}_{\mathrm{N}}=293 \mathrm{~K}$ and $\mathrm{a}=0.426 \mathrm{~nm}$, respectively. In $\mathrm{CoO}$, the spin and orbital moments are known from X-ray magnetic diffraction. From the Co spin moment in $\mathrm{CoO}, \mu_{\mathrm{s}}=2.55 \mu_{\mathrm{B}}$, one derives a spin effective moment $\mu_{\text {eff }}=3.40 \mu_{\mathrm{B}}$. Thus $w+w^{\prime}=320$. Surface atoms have 9 neighbours instead of 12, assuming that the molecular field coefficients are reduced in proportion, one obtains: $w_{\text {surf }}+w_{\text {surf }}^{\prime}=240$. We arbitrarily assumed $w=w^{\prime}$. Thus $w_{\text {surf }}=w_{\text {surf }}=120$. Assuming that only surface atoms are canted under the effect of $H_{\text {exch }}$ and with $\mathrm{w}=\mathrm{w}$, the exchange energy arising from the coupling with the FM moments is $5 / 3 w M \theta^{2}$ instead of $2 w M \theta^{2}$ for a usual antiferromagnet ( $\theta$ is the canting angle induced by the FM exchange field on the interface Co atoms of the CoO AFM matrix). Thus, the coefficient to be taken in the usual expression of the susceptibility is $120 \times 5 / 6=100$.

\section{Appendix C. Coherent rotation}

At small particle sizes, coherent rotation is favoured since the domain wall energy, a surface energy term, becomes larger than the Zeeman energy, a volume energy term. Recent experimental measurements on Co nanoparticles have experimentally demonstrated the occurrence of coherent rotation [22]. In the present case, the situation is different since the Co nanoparticles are coupled to the antiferromagnetic matrix and we may assume that a domain wall forms within the matrix during reversal. The domain wall energy within the AFM matrix is estimated to be $\gamma_{\text {wall }} \approx 510^{-2} \mathrm{~J} / \mathrm{m}^{2}\left(A=5.510^{-12} \mathrm{~J} / \mathrm{m}\right.$ and $\mathrm{K}_{1}$ $=2.710^{7} \mathrm{~J} / \mathrm{m}^{3}$ are assumed). Assuming that the wall surface area equals the nanoparticle surface area, one obtains $E_{\text {wall }}$ $=3.710^{-18} \mathrm{~J}$. This energy is much larger than the experimental exchange bias energy which is given by the Zeeman energy, $210^{-19} \mathrm{~J}$, or than the calculated energy barrier for coherent rotation which is $3.410^{-19} \mathrm{~J}$. Hence, it can be concluded that reversal necessarily occurs by coherent rotation.

\section{Aknowledgments}

Financial support from the NEXBIAS (HPRN-CT 200200296), the 2001-SGR-00189 and the MAT-2004-01679 research projects is acknowledged. 


\section{References}

[1] R.H. Kodama, J. Magn. Magn. Mater. 200 (1999) 359.

[2] D. Weller, A. Moser, IEEE Trans. Magn. 35 (1999) 4423.

[3] V. Skumryev, S. Stoyanov, Y. Zhang, G. Hadjipanayis, D. Givord, J. Nogués, Nature 423 (2003) 850.

[4] K. Liu, J. Nogués, C. Leighton, H. Masuda, K. Nishio, I. V. Roshchin, I. K. Schuller Appl. Phys. Lett. 81, (2002) 4434.

[5] C. Frandsen, C. W. Ostenfeld, M. Xu, C. S. Jacobsen, L. Keller, K. Lefmann, S. Morup, Phys.Rev. B 70 (2004) 134416

[6] S. Stoyanov, V. Skumryev, Y. Zhang, Y. Huang, G Hadjipanayis, J. Nogués, J. Appl. Phys. 93 (2003) 7592.

[7] J. Eisenmenger, I. K. Schuller, Nature Materials 2 (2003) 437.

[8] J. Nogués, J. Sort V. Langlais, S. Doppiu, B. Dieny, J.S. Muñoz, S. Suriñach, M.D. Baró, S. Stoyanov, Y. Zhang, Int. J. Nanotechnology (2005) in press.

[9] P. J. Jensen, Appl. Phys. Lett. 78 (2001) 2190.

[10] J. Mejía-López, D. Altbir, and I.K. Schuller,Appl. Phys. Lett. 83, 332 (2003).

[11] J. Nogués, I. K. Schuller, J. Magn. Magn. Mater. 192 (1999) 203.

[12] W. H. Meiklejohn, C. P. Bean, Phys. Rev. 105 (1957) 904

[13] K. Takano, R. H. Kodama, A. E. Berkowitz, W Cao, G. Thomas, Phys. Rev. Lett. 79 (1997) 1130

[14]Landolt-Börnstein, Numerical data and functional relationships in Science and Technology (Springer-Verlag, Berlin, 1975) 7, II.1.1

[15] N. C. Koon, Phys. Rev. Lett. (1997), 78(25), 4865

[16] T. C. Schulthess, W. H. Butler, Phys. Rev. Lett. 81, (1998) 4516.

[17] R. L. Stamps, J. Phys. D: Appl. Phys. 33 (2000) R247.

[18] S. Chikazumi, Physics of Ferromagnetism, (Oxford University Press, New York, 1997).

[19] J. Kanamori, Prog. Theor. Phys. 17 (1957) 177.

[20] R. H. Kodama, S. H. Makhlouf, A. E. Berkowitz, Phys. Rev. Lett. 79 (1997) 1393.

[21] J. M. D. Coey, Phys. Rev. Lett. 27 (1971) 1140.

[22] M. Jamet, W. Wernsdorfer, C. Thirion, D. Mailly, V. Dupuis, P. Melinon, A. Perez, Phys. Rev. Lett., 86 (2001) 4588.

\section{Figure captions}

Fig.1. Possible coupling schemes for magnetic moments of Co nanoparticles embedded in $\mathrm{CoO}$ : (a) uncompensated case - parallel coupling; (b) compensated case perpendicular coupling.

Fig.2. Possible schemes for magnetization reversal: (a) initial state; (b) magnetization rotation within the AFM easy plane; (c) magnetization rotation in the plane perpendicular to the AFM easy axis; (d) final state. 